\title{
The Neighborhood Characteristic Parameter for Graphs
}

\author{
Terry A. McKee \\ Department of Mathematics \& Statistics \\ Wright State University, Dayton, Ohio 45435, USA \\ terry.mckee@wright.edu
}

Submitted: Aug 19, 2001; Accepted: May 5, 2003; Published: May 7, 2003

MR Subject Classifications: 05C75, 05C99

\begin{abstract}
Define the neighborhood characteristic of a graph to be $s_{1}-s_{2}+s_{3}-\cdots$, where $s_{i}$ counts subsets of $i$ vertices that are all adjacent to some vertex outside the subset. This amounts to replacing cliques by neighborhoods in the traditional 'Euler characteristic' (the number of vertices, minus the number of edges, plus the number of triangles, etc.). The neighborhood characteristic can also be calculated by knowing, for all $i, j \geq 2$, how many $K_{i, j}$ subgraphs there are or, through an Euler-Poincarétype theorem, by knowing how those subgraphs are arranged. Chordal bipartite graphs are precisely the graphs for which every nontrivial connected induced subgraph has neighborhood characteristic 2.
\end{abstract}

\section{The Neighborhood Characteristic}

Define the neighborhood characteristic of any graph $G$ without isolated vertices to be

$$
\operatorname{schar}(G)=s_{1}-s_{2}+s_{3}-\cdots,
$$

where $s_{i}$ is the number of subsets of $V(G)$ of cardinality $i$ that are externally dominated, meaning that $S \subseteq N(v)$ for some $v \in V(G)-S$. Thus $s_{1}=n$ is just the order of $G$, and $s_{2}$ is the number of pairs of vertices that have a common neighbor.

For comparison, the traditional (Euler) characteristic [7] — which might be thought of as the clique characteristic - is

$$
\operatorname{char}(G)=k_{1}-k_{2}+k_{3}-\cdots,
$$

where $k_{i}$ is the number of complete subgraphs of $G$ of order $i$; thus $k_{1}=s_{1}, k_{2}=m$ is the number of edges, and $k_{3}$ is the number of triangles. So schar $(G)$ can be thought of as modifying $\operatorname{char}(G)$ by replacing complete subgraphs with externally dominated subgraphs. In 
topological terms, $\operatorname{char}(G)$ is the characteristic of the simplicial complex whose simplices are the complete subgraphs of $G$, and ${ }_{\mathcal{N}} \operatorname{char}(G)$ is the characteristic of the "neighborhood complex' $\mathcal{N}(G)$, as in [1], whose simplices are the externally dominated subgraphs of $G$.

\section{Simple Examples:}

$$
\begin{aligned}
& \mathcal{N c h a r}\left(C_{n}\right)= \begin{cases}4-2=2 & \text { if } n=4 \\
n-n=0 & \text { if } n \neq 4\end{cases} \\
& \mathcal{N} \operatorname{char}\left(K_{n}\right)=n-\left(\begin{array}{l}
n \\
2
\end{array}\right)+\cdots+(-1)^{n}\left(\begin{array}{c}
n \\
n-1
\end{array}\right)= \begin{cases}2 & \text { if } n \text { is even } \\
0 & \text { if } n \text { is odd }\end{cases} \\
& \mathcal{N} \operatorname{char}\left(K_{m, n}\right)=[m+n]-\left[\left(\begin{array}{c}
m \\
2
\end{array}\right)+\left(\begin{array}{c}
n \\
2
\end{array}\right)\right]+\left[\left(\begin{array}{c}
m \\
3
\end{array}\right)+\left(\begin{array}{l}
n \\
3
\end{array}\right)\right]-\cdots=2 \\
& \mathcal{N} \operatorname{char}\left(C_{n}+K_{1}\right)= \begin{cases}5-10+6-1=0 \\
(n+1)-\left(\begin{array}{c}
n+1 \\
2
\end{array}\right)+\left[\left(\begin{array}{l}
n \\
3
\end{array}\right)+n\right]-\left(\begin{array}{c}
n \\
4
\end{array}\right)+\cdots=2 \text { if } n \neq 4\end{cases} \\
& \mathcal{N} \operatorname{char}(\text { cube })=8-12+8=4 \\
& \mathcal{N} \operatorname{char}(\text { octahedron })=6-15+12-3=0 \\
& \mathcal{N} \operatorname{char}(\text { dodecahedron })=20-60+20=-20
\end{aligned}
$$

\section{Computing the Neighborhood Characteristic}

The neighborhood characteristic of a graph can also be calculated in terms of the complete bipartite subgraphs present in $G$. Let $k_{i, j}$ count the number of complete bipartite-but not necessarily induced - subgraphs that are isomorphic to $K_{i, j}\left(\right.$ so $\left.k_{i, j}=k_{j, i}\right)$. Notice that $s_{i}$ is not necessarily equal to $k_{1, i}$ since the same $i$ vertices could be counted in more than one $K_{1, i}$. Such overcounting is corrected for in the following theorem (which also shows that, for a bipartite graph $G, \operatorname{schar}(G)$ equals twice the 'bipartite characteristic' defined in [5]).

Theorem 1 For every graph $G$ without isolated vertices,

$$
\mathcal{N c h a r}(G)=2 \sum_{1 \leq i \leq j}(-1)^{i+j} k_{i, j}
$$

Proof. Using simple counting arguments, noting the symmetry of each $K_{i, i},{ }_{\text {vchar }}(G)=$ $s_{1}-s_{2}+s_{3}-\cdots$ equals

$$
n-\left(\begin{array}{c}
k_{1,2} \\
-2 k_{2,2} \\
+k_{3,2} \\
-k_{4,2} \\
\vdots
\end{array}\right)+\left(\begin{array}{c}
k_{1,3} \\
-k_{2,3} \\
+2 k_{3,3} \\
-k_{4,3} \\
\vdots
\end{array}\right)-\cdots=n+\left(\begin{array}{c}
-k_{1,2}+k_{1,3}-\cdots \\
+2 k_{2,2}-k_{2,3}+\cdots \\
-k_{3,2}+2 k_{3,3}-\cdots \\
\vdots \\
\vdots
\end{array}\right.
$$


which, using $k_{i, j}=k_{j, i}$, can be rewritten as

$$
n-2 k_{1,1}+k_{1,2}-k_{1,3}+\cdots+2\left(\begin{array}{r}
k_{1,1}-k_{1,2}+k_{1,3}-\cdots \\
+k_{2,2}-k_{2,3}+\cdots \\
\\
\\
\\
\\
\\
\\
\end{array}\right.
$$

The final term in (4) equals the right side of (3), while the rest of (4) equals $\sum_{v}\left[\left(\begin{array}{c}\operatorname{deg} v \\ 0\end{array}\right)-\right.$ $\left.\left(\begin{array}{c}\operatorname{deg} v \\ 1\end{array}\right)+\left(\begin{array}{c}\operatorname{deg} v \\ 2\end{array}\right)-\cdots\right]=0$.

Therefore $\operatorname{schar}(G)$ is always even. Moreover, the computation in formula (3) can be reduced to

$$
\mathcal{N} \operatorname{char}(G)=2\left(n-m+\sum_{2 \leq i \leq j}(-1)^{i+j} k_{i, j}\right)
$$

by first noting that expression (4) also equals

$$
n-k_{1,2}+k_{1,3}+\cdots+2\left(\begin{array}{r}
k_{2,2}-k_{2,3}+\cdots \\
+k_{3,3}-\cdots \\
\\
\end{array}\right.
$$

The final term in (6) equals $2 \sum_{2 \leq i \leq j}(-1)^{i+j} k_{i, j}$, while the rest of $(6)$ equals $(2 n-2 m-$ $\left.n+2 k_{1,1}\right)-k_{1,2}+k_{1,3}-\cdots$, which in turn equals

$$
2 n-2 m-\sum_{v}\left[\left(\begin{array}{c}
\operatorname{deg} v \\
0
\end{array}\right)-\left(\begin{array}{c}
\operatorname{deg} v \\
1
\end{array}\right)+\left(\begin{array}{c}
\operatorname{deg} v \\
2
\end{array}\right) \cdots\right]=2(n-m) .
$$

Notice too that, by (5), if $G$ contains no $C_{4}$ subgraphs (induced or not), then $\operatorname{cchar}(G)=$ $2(n-m)$.

A vertex $v$ of $G$ is called covered in [2] if some vertex of $G-v$ externally dominates $N(v)$. The following theorem reduces the calculation of $\operatorname{schar}(G)$ to graphs $G$ with no covered vertices (in other words, to graphs whose open neighborhoods are pairwise incomparable).

Theorem 2 If $v$ is a covered vertex of $G$, then $\operatorname{schar}(G)=\operatorname{schar}(G-v)$.

Proof. Suppose $v$ is a covered vertex of $G$ and $S$ is any subset of $N(v)$ with $|S| \geq 2$ and with $S$ externally dominated by $d \geq 1$ vertices of $G-v$. Vertex $v$ can be involved with part of $N(v)$ in a complete bipartite (not necessarily induced!) subgraph $H+H^{\prime}$-and so contribute to $k_{i, j}$ in expression (5) - in two ways:

CASE 1: $v \in H, S=H^{\prime}$, and $H \cap N(v)=\emptyset$. For each $i \geq 1$, there are $\left(\begin{array}{l}d \\ i\end{array}\right)$ subgraphs isomorphic to $K_{i+1,|S|}$ that involve $v$ and $S$ in this way, so the total contribution to $\operatorname{schar}(G)-{ }_{\mathcal{N}} \operatorname{char}(G-v)$ in expression (5) in this case is

$$
(-1)^{2+|S|}\left(\begin{array}{l}
d \\
1
\end{array}\right)+(-1)^{3+|S|}\left(\begin{array}{l}
d \\
2
\end{array}\right)+\cdots+(-1)^{d+1+|S|}\left(\begin{array}{l}
d \\
d
\end{array}\right)=(-1)^{|S|}
$$


CASE 2: $v \in H, S=H^{\prime} \cup\left\{w_{1}, \ldots, w_{h}\right\}, H \cap N(v)=\left\{w_{1}, \ldots, w_{h}\right\}$, and $h \geq 1$. For each $i \geq 0$, there are $\left(\begin{array}{l}d \\ i\end{array}\right)$ subgraphs isomorphic to $K_{i+1+h,|S|-h}$ that involve $v$ and $S$ in this way, the total contribution to $\operatorname{schar}(G)-{ }_{\mathcal{N}} \operatorname{char}(G-v)$ in expression (5) in this case is

$$
(-1)^{1+|S|}\left(\begin{array}{l}
d \\
0
\end{array}\right)+(-1)^{2+|S|}\left(\begin{array}{l}
d \\
1
\end{array}\right)+\cdots+(-1)^{d+1+|S|}\left(\begin{array}{l}
d \\
d
\end{array}\right)=0 .
$$

Adding $v$ to $G-v$ increases $n$ by 1 and $m$ by $|N(v)|$. Therefore, the total contribution to $\operatorname{schar}(G)-\operatorname{schar}(G-v)$ involving all $S \subseteq N(v)$ in expression (5) is

$$
2\left(1-|N(v)|+\sum_{S \subseteq N(v),|S| \geq 2}(-1)^{|S|}\right)=2 \sum_{i \geq 0}(-1)^{i}\left(\begin{array}{c}
|N(v)| \\
i
\end{array}\right)=0 .
$$

Therefore, $\operatorname{cchar}(G)={ }_{\mathcal{N}} \operatorname{char}(G-v)$.

A chordal bipartite graph is a bipartite graph in which every cycle of length at least six has a chord; see $[6, \S 7.3]$ and the papers cited there. Suppose $G$ is a chordal bipartite graph. In [3], a set $S \subseteq V(G)$ is called a minimal edge separator if there exist edges $e$ and $f$ that are in different components of the subgraph $G-S$ induced by $V(G)-S$, and no proper subset of $S$ has that same property. If $S$ is a minimal edge separator of $G$, with $e$ and $f$ as above, then the definition of chordal bipartite implies that every two vertices in $S$ of opposite 'color' in $G$ will be adjacent (they will be endpoints of a chord in a cycle that contains $e$ and $f$ ). If $S$ is an edge separator of $G$ with one component of $G-S$ as small as possible, then $S$ will contain an edge $e$ with endpoints $v$ and $w$ such that every two vertices in $N(v) \cup N(w)$ of opposite color in $G$ will be adjacent. Such an edge is called a bisimplicial edge. As in [3], this shows that every chordal bipartite graph contains a bisimplicial edge.

The following corollary is analogous to the observation in [4] that a graph is chordal if and only every induced subgraph $H$ has $\operatorname{char}(H)=1$. (Notice that the proof shows that $\operatorname{schar}(H)=2$ in the statement of the corollary could be equivalently replaced by $\operatorname{schar}(H) \neq 0$.)

Corollary 3 A graph with no isolated vertices is chordal bipartite if and only if every connected induced subgraph $H$ of order $\geq 2$ has $\operatorname{schar}(H)=2$.

Proof. First suppose $G$ is a chordal bipartite graph and $H$ is any connected induced subgraph of $G$ with $|V(H)| \geq 2$. Then $H$ must be chordal bipartite as well. Since $G$ is chordal bipartite, there will be a bisimplicial edge $v w$ in $G$ and, without loss of generality, $v$ can be assumed to have degree at least two. Then $w$ is covered and can be removed with, by Theorem $2, \operatorname{schar}(H)=\operatorname{schar}(H-w)$. Repeating this eventually ends with a single edge, and so $\mathrm{schar}(H)=2$.

Conversely, suppose $G$ is not chordal bipartite. If $G$ is not bipartite, then $G$ contains an induced odd cycle $C$ and ${ }_{\text {schar }}(C)=0$. If $G$ is bipartite but not chordal bipartite, then $G$ must contain an induced even cycle $C$ of length at least six, and again $\operatorname{schar}(C)=0$. 


\section{An Euler-Poincaré-type Theorem}

This section develops machinery for the Euler-Poincaré-like Theorem 4, a formula for calculating cchar $(G)$ in terms of, roughly speaking, the arrangement of the $K_{i, j}$ subgraphs present in $G$. This development parallels [7].

For any graph $G$, define the 0-dimensional bicliques to be the vertices of $G$, the 1dimensional bicliques to be the edges, the 2-dimensional bicliques to be all the $K_{2,2}$ subgraphs (where 'all' means 'whether induced or not'), the 3-dimensional bicliques to be all the $K_{2,3}$ subgraphs, and for $j \geq 4$, the $j$-dimensional bicliques to be all the $K_{i, j-i+2}$ subgraphs for which $2 \leq i<j$.

Define the boundary of a $j$-dimensional biclique to be the set of all the $(j-1)$ dimensional bicliques it contains (or the empty set when $j=0$ ). Thus the boundary of an edge consists of its two endpoints, the boundary of a $K_{2,2}$ consists of the four edges of that 4-cycle, the boundary of a $K_{2,3}$ consists of its three 4-cycles, and so on. The boundary of a set $\left\{S_{1}, \ldots, S_{\ell}\right\}$ of $j$-dimensional bicliques is the symmetric difference of the boundaries of the $S_{i}$ 's. Thus the boundary of the edge set of a path consists of the endpoints of the path, while the boundary of the edge set of a cycle is empty. The boundary of the set of 4-cycles of a cube is also empty, as is the boundary of any set of vertices.

For $j \geq 1$, define a $j$-N circuit to be any set $\mathcal{S}$ of $j$-dimensional bicliques whose boundary is empty. For instance, the edge set of all cycles in a graph is a 1-scircuit, and the six 4-cycles of a cube form a 2-scircuit, as do the $n$ 4-cycles of any wheel $C_{n}+K_{1}$ with $n \neq 4$. In $C_{4}+K_{1}$, let $A, B$, and $C$ be the 4-cycles contained in one of the $K_{2,3}$ subgraphs and $C, D$, and $E$ be the 4-cycles contained in the other $K_{2,3}$ subgraph. Then $\{A, B, C\}$, $\{C, D, E\}$, and $\{A, B, D, E\}$ are 2-scircuits. The six $K_{2,3}$ subgraphs in a $K_{3,3}$ form a 3 -scircuit, but wheels have no 3 -scircuits. The set of all $j$-scircuits of a graph forms a vector space over $\mathbb{Z}_{2}$, with an empty $j$-scircuit as the zero vector, $1 \mathcal{S}=\mathcal{S}$ and $0 \mathcal{S}=0$ defining scalar multiplication, and the sum of $j$-scircuits being the symmetric difference of their sets of $j$-dimensional bicliques.

Call two $j$-scircuits bihomologous whenever either is the sum of the other along with any number of $(j+1)$-dimensional bicliques - or, equivalently, if their sum is the boundary of some set of $(j+1)$-dimensional bicliques. When $j=1$ for instance, two cycles (1scircuits) are bihomologous if one is the sum of the other and 4-cycles. Thus, all cycles of an cube are pairwise bihomologous, as are all the triangles of a wheel, and as are all the 4-cycles of a wheel. When $j=2$, using the notation in the preceding paragraph, the 2-scircuit $\{A, B, D, E\}$ of $C_{4}+K_{1}$ is bihomologous to the empty 2-scircuit (using the two 3 -dimensional bicliques), as is each of the 2-scircuits $\{A, B, C\}$ and $\{C, D, E\}$ (automatically, since each is itself a 3-dimensional biclique). The 2 -scircuit of $K_{3,3}$ that consists of all nine 4-cycles is also bihomologous to the empty 2-scircuit (using the three 3-dimensional bicliques $\left[K_{2,3} \mathrm{~s}\right]$ that contain all the vertices of either of the two color classes).

For any $j$-scircuit $\mathcal{S}$, let $[\mathcal{S}]$ denote its bihomology class - the equivalence class of $j$ scircuits bihomologous to $\mathcal{S}$. The bihomology classes of all $j$-scircuits of $G$ form another $\mathbb{Z}_{2}$-vector space where the zero vector is the bihomology class of the empty $j$-scircuit 
and the sum of bihomology classes $\left[\mathcal{S}_{1}\right]$ and $\left[\mathcal{S}_{2}\right]$ is the bihomology class of the sum (symmetric difference) of $\mathcal{S}_{1}$ and $\mathcal{S}_{2}$. Let $\beta_{j}^{\mathcal{N}}(G)$ denote the dimension of the vector space of bihomology classes of $j$-scircuits of $G$. For $j=0$, there is a basis consisting of one representative vertex from each component, and so $\beta_{0}^{\mathcal{N}}(G)$ is the number of components of $G$. For $j=1$, there is a basis consisting of selected cycles with lengths different from four, with $\beta_{1}^{\mathcal{N}}(G)=0$ if and only if the circuit space of $G$ has a basis consisting of 4-cycles. For instance, the cube has $\beta_{0}^{\mathcal{N}}=1$ since it is connected, $\beta_{1}^{\mathcal{N}}=0$ since the circuit space has a basis consisting of (any five) 4-cycles, $\beta_{2}^{\mathcal{N}}=1$ since the six 4-cycles form the only 2-scircuit (there are no 3-dimensional bicliques), and $\beta_{i}^{\mathcal{N}}=0$ for all $i \geq 3$ since there are no such $i$-scircuits. Similarly, $C_{4}+K_{1}$ has $\beta_{0}^{\mathcal{N}}=\beta_{1}^{\mathcal{N}}=1$ and $\beta_{i}^{\mathcal{N}}=0$ for all $i \geq 2$; all the other wheels are the same except that $\beta_{2}^{\mathcal{N}}=1$.

Theorem 4 is the ${ }_{\text {schar }}(G)$ analogy of the Euler-Poincaré theorem [7] for $\operatorname{char}(G)$. To illustrate formula $(7)$, the cube has ${ }_{\text {schar }}=2(1-0+1-0+\cdots)=4, \operatorname{schar}\left(C_{4}\right)=$ $2(1-0+0+\cdots)=2$, and $\operatorname{schar}\left(C_{4}+K_{1}\right)=2(1-1+0+\cdots)=0 ;$ when $n \neq 4$, $\operatorname{schar}\left(C_{n}\right)=2(1-1+0+\cdots)=0$ and $\operatorname{schar}\left(C_{n}+K_{1}\right)=2(1-1+1-0+0+\cdots)=2$.

Theorem 4 For every graph $G$ without isolated vertices,

$$
\mathcal{N} \operatorname{char}(G)=2\left(\beta_{0}^{\mathcal{N}}-\beta_{1}^{\mathcal{N}}+\beta_{2}^{\mathcal{N}}-\cdots\right) .
$$

Proof. For every integer $j \geq 0$, let $B_{j}$ be the set of all sets $\mathcal{S}$ of $j$-dimensional bicliques of $G$. As with any power set, each $B_{j}$ is a vector space over $\mathbb{Z}_{2}$ with symmetric difference as sum. Since the singletons of $B_{j}$ form a standard basis, $\operatorname{dim}\left(B_{0}\right)=n, \operatorname{dim}\left(B_{1}\right)=m$, $\operatorname{dim}\left(B_{2}\right)=k_{2,2}, \operatorname{dim}\left(B_{3}\right)=k_{2,3}$, and for $j \geq 4, \operatorname{dim}\left(B_{j}\right)=\sum_{i} k_{i, j-i+2}$ over all $i$ for which $2 \leq i<j$.

For each $j \geq 1$, taking boundaries of members of $B_{j}$ constitutes a map $\partial_{j}: B_{j} \rightarrow B_{j-1}$ between vector spaces. A set $\mathcal{S}$ of $j$-dimensional bicliques is a $j$-scircuit if and only if $\mathcal{S} \in \operatorname{Kernel}\left(\partial_{j}\right)$, whereas $\mathcal{S}$ is a boundary of a set of $(j+1)$-dimensional bicliques if and only if $\mathcal{S} \in \operatorname{Image}\left(\partial_{j+1}\right)$.

Since the vector space of bihomology classes of $j$-scircuits of $G$ is formed from $j$ Ncircuits modulo the boundaries of $(j+1)$-dimensional bicliques, this vector space is isomorphic to the quotient space $\operatorname{Kernel}\left(\partial_{j}\right) / \operatorname{Image}\left(\partial_{j+1}\right)$ and so has dimension $\beta_{j}^{\mathcal{N}}=$ $\operatorname{dim}\left(\operatorname{Kernel}\left(\partial_{j}\right)\right)-\operatorname{rank}\left(\partial_{j+1}\right)$. But

$$
\operatorname{dim}\left(\operatorname{Kernel}\left(\partial_{j}\right)\right)+\operatorname{rank}\left(\partial_{j}\right)=\operatorname{dim}\left(B_{j}\right)
$$

by the dimension theorem for vector spaces, so

$$
\beta_{j}^{\mathcal{N}}=\operatorname{dim}\left(B_{j}\right)-\operatorname{rank}\left(\partial_{j}\right)-\operatorname{rank}\left(\partial_{j+1}\right) .
$$

Since $\beta_{0}^{\mathcal{N}}$ counts the number of connected components of $G, \operatorname{Kernel}\left(\partial_{1}\right)$ (because it is the 'circuit subspace' of $G$; see [8]) has dimension $m-n+\beta_{0}^{\mathcal{N}}$ (the 'cyclomatic number' of $G$ ). So by $(8), \operatorname{rank}\left(\partial_{1}\right)=m-\left[m-n+\beta_{0}^{\mathcal{N}}\right]=n-\beta_{0}^{\mathcal{N}}$. For $j \geq \max \left\{i: s_{i} \neq 0\right\}$, 
$\operatorname{rank}\left(\partial_{j+1}\right)=0$. Therefore, using $(9)$,

$$
\begin{aligned}
\sum_{j \geq 0}(-1)^{j} \beta_{j}^{\mathcal{N}} & =\beta_{0}^{\mathcal{N}}+\sum_{j \geq 1}(-1)^{j}\left[\operatorname{dim}\left(B_{j}\right)-\operatorname{rank}\left(\partial_{j}\right)-\operatorname{rank}\left(\partial_{j+1}\right)\right] \\
& =\beta_{0}^{\mathcal{N}}+\operatorname{rank}\left(\partial_{1}\right)+\sum_{j \geq 1}(-1)^{j} \operatorname{dim}\left(B_{j}\right) \\
& =\beta_{0}^{\mathcal{N}}+\left(n-\beta_{0}^{\mathcal{N}}\right)-m+k_{2,2}+\sum_{j \geq 3}(-1)^{j} \operatorname{dim}\left(B_{j}\right) \\
& =n-m+k_{2,2}+\sum_{j \geq 3}(-1)^{j}\left(\sum_{i \geq 2}^{j-1} k_{i, j-i+2}\right) \\
& =n-m+\sum_{2 \leq i \leq j}(-1)^{i+j} k_{i, j}
\end{aligned}
$$

which equals $\frac{1}{2} \mathcal{N} \operatorname{char}(G)$ by $(5)$.

Acknowledgement. The author is grateful for helpful conversations with Erich Prisner.

\section{References}

[1] A. Björner, Combinatorics and topology, Notices Amer. Math. Soc. 32 (1985) 339-345.

[2] F. F. Dragan and V. I. Voloshin, Incidence graphs of biacyclic hypergraphs, Discrete Appl. Math. 68 (1996) 259-266.

[3] M. C. Golumbic and C. F. Goss, Perfect elimination and chordal bipartite graphs, J. Graph Theory 2 (1978) 155-163.

[4] T. A. McKee, How chordal graphs work, Bull. Inst. Combin. Appl. 9 (1993) 27-39.

[5] T. A. McKee, A characteristic approach to bipartite graphs as incidence graphs, Discrete Math., to appear.

[6] T. A. McKee and F. R. McMorris, Topics in Intersection Graph Theory, Society for Industrial and Applied Mathematics, Philadelphia, 1999.

[7] T. A. McKee and E. Prisner, An approach to graph-theoretic homology, in: Y. Alavi, D. R. Lick, and A. Schwenk, eds., Combinatorics, Graph Theory, and Algorithms, Vol 2, New Issues Press, Kalamazoo, MI, 1999, pp. 631-640.

[8] R. J. Wilson, Introduction to Graph Theory, Longman, New York-London, 1996. 